\title{
Was man bei V. a. Schlaganfall (keinesfalls) tun sollte
}

\begin{abstract}
Vor jeglicher spezieller Therapie muss mittels CT geklärt werden, ob einem Schlaganfall eine Hirnblutung oder eine Ischämie zugrunde liegt. Im Vorfeld muss man sich darauf beschränken, eine adäquate Oxygenierung, Normoglykämie und Normothermie anzustreben. Unbedingt zu vermeiden sind ASS und Heparin sowie eine zu starke Senkung des Blutdrucks.
\end{abstract}

— „Wer Heparin vor der CT-Diagnostik gibt, hat eine etwa $15 \%$ ige Chance, den Patienten sofort umzubringen", betonte PD Dr. Martin Köhrmann, Erlangen. Denn so hoch liegt der Anteil von Hirnblutungen. Auch bei den restlichen $85 \%$ der Patienten hilft Heparin nicht, verhindert aber die einzige nachweislich wirksame Therapie, die Thrombolyse.

\section{Blutdruckschwankungen vermeiden}

Über 70\% der Patienten mit akutem Schlaganfall weisen einen erhöhten Blutdruck auf, zum einen wegen einer vorbestehenden Hypertonie, zum anderen, weil sich ein Bedarfsdruck entwickelt hat. Denn in den ischämischen Arealen ist die zerebrale Autoregulation aufgehoben, und die Perfusion hängt somit direkt vom systemischen Blutdruck ab. Dass eine akute Senkung des Blutdrucks das Outcome verschlechtert, zeigten bereits in den 1990er-Jahren Studien mit Nimodipin. Die CHIPPSStudie hat dies aktuell bestätigt. Hier hatten 179 Patienten ACE-Hemmer, Betablocker oder Placebo erhalten.

Die DGN-Leitlinien empfehlen deshalb, den Blutdruck in der Akutphase eines Schlaganfalls nicht zu senken, solange er nicht massiv erhöht ist $(>220$ $\mathrm{mmHg}$ ), sondern ihn im leicht hypertensiven Bereich zu halten. Starke Blutdruckabfälle und hypotone Werte müssen vermieden werden.

\section{Thrombolyse auch im Alltag effektiv und sicher}

Ist die Ischämie bestätigt, kann eine Thrombolyse im 3-Stunden-Zeitfenster signifikant mehr Patienten ein Überle- ben ohne Behinderung ermöglichen, wie die NINDS-Studie bereits Mitte der 1990er-Jahre zeigen konnte. In den USA wurde rtPA aufgrund dieser Ergebnisse sofort zugelassen. Die EMEA erteilte 2002 eine vorläufige Zulassung unter zwei Voraussetzungen: Es sollte durch die Registerstudie SITS-MOST geklärt werden, ob diese Therapie auch in der klinischen Routine funktioniert. Außerdem sollte in ECASS-III der Nutzen von rtPA in einem Zeitfenster von

\section{Subakuter Hirninfarkt}

\section{Fatale Komplikation} nach Heparingabe

Selbst in der frühen Sekundärprophylaxe bei Vorhofflimmern hat Heparin nichts verloren. Es vermindert zwar die frühe Reinfarktrate um etwa $2 \%$, erhöht aber die Blutungsrate um $2 \%$. Netto kommt also rein rechnerisch kein Nutzen heraus. Tatsächlich ist das Gegenteil der Fall. Denn Blutungen unter Antikoagulation verlaufen klinisch besonders schwerwiegend, oft fatal. Köhrmann verdeutlichte dies an einem tragischen Fallbeispiel: Ein 68-Jähriger hatte seit einem Tag eine Beinparese rechts und stellte sich mit dieser relativ leichten Symptomatik im Krankenhaus vor. Dort wurde ein Vorhofflimmern diagnostiziert. Das kraniale CT zeigte einen subakuten Anterior-Infarkt. Wegen des Vorhofflimmerns erhielt der Patient einen Heparinbolus. Etwa zwölf Stunden später lag er komatös im Bett, weil sich aus dem Infarkt eine massive Blutung entwickelt hatte, die schließlich zum Tod führte.
3-4,5 Stunden nach dem Schlaganfall untersucht werden.

SITS-MOST schloss 6483 Patienten ein, die streng innerhalb des 3-Stunden-Fensters thrombolysiert wurden. Von den teilnehmenden 285 europäischen Zentren hatte die Hälfte wenig Vorerfahrung mit Thrombolyse. Trotzdem erwies sich die Lyse in dieser Studie als ebenso effektiv und sicher wie in klinischen Studien.

In ECASS-III wurden 821 Patienten randomisiert, die Alteplase oder Placebo 3-4,5 Stunden nach einem ischämischen Schlaganfall erhielten. Die Ergebnisse bestätigten frühere Daten, dass auch innerhalb von 3-4,5 Stunden ein signifikanter Vorteil im neurologischen Ergebnis zu erzielen ist.

\section{Keine Lizenz zum Trödeln}

Natürlich ist der Gewinn umso größer, je früher interveniert wird. Nach einem Schlaganfall gehen immerhin in einer Minute zwei Millionen Nervenzellen, 14 Milliarden Synapsen und $12 \mathrm{~km}$ myelinisierte Nervenfasern zugrunde! Die ECASS-III-Ergebnisse sind deshalb nicht als Lizenz zum Trödeln zu verstehen. Vielmehr erlauben sie, auch Patienten, für die sich das 3-Stunden-Fenster schon geschlossen hat, wenn sie das Krankenhaus erreichen, von der Thrombolyse profitieren zu lassen. „Es mag also mehr Zeit für die Patienten geben, aber nicht für die behandelnden Ärzte", so Köhrmann. Die Door-to-needle-Zeit sollte deutlich unter einer Stunde liegen.

DR. MED. ANGELIKA BISCHOFF

- 116. Kongress der Deutschen Gesellschaft für Innere Medizin in Wiesbaden 